\title{
Benefits in Speech Recognition in Noise with Remote Wireless Microphones in Group Settings
}

\author{
Linda M. Thibodeau ${ }^{1}$ \\ ${ }^{1}$ Callier Center for Communication Disorders, University of Texas at \\ Dallas, Dallas, TX \\ J Am Acad Audiol 2020;31:404-411.
}

\begin{abstract}
Address for correspondence Linda M. Thibodeau, Callier Center for Communication Disorders, University of Texas at Dallas, Dallas, TX 75235 (e-mail: thib@utdallas.edu).
\end{abstract}

\begin{abstract}
Background Although hearing aids (HAs) and cochlear implants ( $\mathrm{Cls}$ ) can provide significant benefits to persons with hearing loss, users frequently report difficulty hearing in noisy environments, particularly when there are multiple talkers. Little is known about the benefits provided by currently available wireless microphones in multitalker situations.

Purpose The purpose of this study was to compare the benefits received in speech recognition in noise by adults with hearing loss when using two different wireless microphone types in a simulated group setting.

Research Design A quasi-experimental, repeated-measures design was used where performance in a control condition, $\mathrm{HA} / \mathrm{Cl}$ alone, was compared with performance in two wireless microphone intervention conditions.

Study Sample Participants included ten listeners, aged 20-92 years, with bilateral sensorineural hearing loss who were experienced $\mathrm{HA}$ or $\mathrm{Cl}$ users.

Intervention The two wireless microphones by Phonak, Roger Pen, and Roger Select used the same digital modulation protocol to transmit the signal to compatible receivers. However, the Roger Pen operated in a fixed omnidirectional mode, whereas the Roger Select operated in an adaptive directional mode.

Data Collection and Analysis Participants were asked to repeat Hearing in Noise Test sentences presented in restaurant noise in three conditions: $\mathrm{HA} / \mathrm{Cl}$ alone, $\mathrm{HA} / \mathrm{Cl}$ with a Roger Pen, or $\mathrm{HA} / \mathrm{Cl}$ with a Roger Select microphone placed in the center of a round table. Sentences were presented from one of five loudspeakers equally spaced with the participant, while restaurant noise was presented on each side at four signal-to-noise ratios (SNRs), including $+5,0,-5$, and $-10 \mathrm{~dB}$. A two-way, repeated-measures analysis of variance was performed with main effects of listening condition and noise level.

Results Significantly greater speech recognition performance was achieved with the wireless microphones than with listening with just the $\mathrm{HA}$ or $\mathrm{Cl}$. Furthermore, at the -5 - and $-10-d B$ SNR conditions, the Roger Select resulted in significantly better performance than the Roger Pen microphone.

\section{Keywords}

- hearing loss

- speech perception

- wireless technology

Conclusions The results suggest that the Roger Select microphone can provide significant benefits in speech recognition in noise over the use of $\mathrm{HA} / \mathrm{Cl}$ alone $(61 \%)$ and also significant benefits over the use of a Roger Pen (16\%) in a simulated group dining experience.
\end{abstract}

Copyright $\odot 2020$ by the American Academy of Audiology. All rights reserved. Thieme Medical Publishers, Inc., 333 Seventh Avenue, New York, NY 10001 , USA. Tel: $+1(212) 760-0888$.
DOI https://doi.org/ 10.3766/jaaa. 19060. ISSN 1050-0545. 


\section{Introduction}

Persons with hearing loss experience significant speech recognition deficits when listening in noisy environments despite sophisticated ear-level technology. This is true for persons with cochlear implants (CIs) and amplification. One solution has been to improve the signal-to-noise ratio (SNR) by using wireless technology that will interface with the earlevel device. The benefits of wireless microphone technology when measured with a single talker have been well established (Boothroyd ${ }^{3}$; Lewis et al ${ }^{10}$; Thibodeau ${ }^{14,15}$ ). A wireless microphone system is now even included as a standard component dispensed with one CI system.

The research has focused primarily on educational benefits dating back to 1984 when Hawkins ${ }^{7}$ showed significant gains in speech recognition in nine students with behindthe-ear HAs across various FM coupling options, including direct audio input, neckloop, and silhouette arrangements. The greatest benefit, a $15-\mathrm{dB}$ improvement in the SNR necessary for $50 \%$ correct identification of spondees presented from a single speaker, was obtained when the system was set to a FM receiver only rather than a FM 1 HA microphone. Significant benefit was also observed with directional microphones in both the HAs and the transmitter microphone compared with omnidirectional microphones.

Despite significant technological advances in personal assistive devices with digital noise reduction and adaptive microphone patterns in ear-level technology, users still report dissatisfaction in noise, particularly when there are multiple speakers $\left(\operatorname{Kochkin}^{8}\right)$. Perhaps the most common group communication challenge for adults is dining in a typical noisy restaurant setting. There are currently several options to consider for this situation, which include small, clip-on microphones or more sophisticated, larger devices with multidirectional microphones. These may involve one or more transmission protocols, such as FM (217 MHz), standard Bluetooth $(2.4 \mathrm{GHz})$, or proprietary digital streaming $(2.4 \mathrm{GHz})$. The smaller clip-on options typically involve a single microphone and may work well when there are only two persons dining. There could be some drawbacks if the clip-on microphone is so close to the talker's mouth that unwanted sounds associated with eating are transmitted. However, individuals with HAs or CIs experience significant challenges when dining with groups of friends because different persons speak at random times. The more sophisticated wireless microphones by Phonak, such as the Roger Clip-On or Roger Pen, use proprietary digital streaming via $2.4 \mathrm{GHz}$ and can be set up in a network so that each person at a table could transmit to a person with hearing challenges. Having a Roger Pen or Clip-on microphone on each guest is a somewhat costly arrangement, and the person with hearing loss has no control over the microphones on each talker. The input from neighboring individuals who have side conversations may be quite annoying if there is no agreement among all guests for one person to speak at a time.

One solution that would allow access to multiple talkers and the ability to select the desired conversations around a table would be to use a multimicrophone disc with convenient touch-sensitive microphone activation. Phonak has recently developed the Roger Select microphone, based on the same proprietary digital streaming $2.4-\mathrm{GHz}$ protocol as the Roger Clip-on and Pen. Although designed for group conversations, it can be used by a single talker (Gigandet et $\mathrm{al}^{6}$ ). This two-inch circular device consists of three omnidirectional microphones arranged in a triangle to create six different beams of sensitivity to pick up voices from around a table. It can also be worn on the chest and then would have a directional pattern to focus on the talker's voice. The multibeam technology automatically selects the optimal beam to activate based on the assumption that the greatest SNR is coming from the talker of interest. Although the input is analyzed hundreds of times per second, the result may not always be a desired signal, and therefore, the user may deactivate certain beams via a quick finger tap to focus on talkers of interest.

De Ceulaer et $\mathrm{al}^{5}$ recognized the need to improve communication in group settings and evaluated the benefit of the Roger Pen transmitter microphone in a multitalker network with CI users. Using the Naida Q70 CI and Phonak-integrated Roger 17 receivers, 12 adults repeated sentences from a Flemish sentences-in-noise test presented randomly from one of three speakers to simulate a small group conversation in multitalker babble noise. The speech reception threshold was determined in three conditions: implant alone, Roger Pen placed in the center of the three speakers, and three Roger Pens in a multitalker network. The network of three Roger Pens provided a significant advantage over one Roger Pen for four of the six SNRs tested. The benefits of using a Roger Pen in group settings were also reported by Land and Thibodeau ${ }^{9}$ who found an average improvement in sentence recognition of $18 \%$ compared with listening with HAs alone.

The new Roger Select microphone would potentially provide a more feasible and economic solution than using multiple Roger Pens. To evaluate the benefit of the Roger Select microphone in a group setting, speech recognition in noise using a five-talker speaker array was measured in three conditions: hearing aid or $\mathrm{CI}(\mathrm{HA} / \mathrm{CI})$ alone, $\mathrm{HA} / \mathrm{CI}+$ Roger Pen, and $\mathrm{HA} / \mathrm{CI}+$ Roger Select. Specifically, the purpose of this study was to determine if sentence recognition in noise is significantly better for participants with impaired hearing who use a Roger Select wireless system than for those who use a Roger Pen or no wireless microphone when the signal is coming from multiple locations in a simulated restaurant arrangement. Because Thibodeau ${ }^{15}$ reported that the benefits obtained with Roger technology were greater at higher noise levels, it was of interest to explore the wireless microphone benefits across a range of noise levels.

\section{Method}

\section{Participants}

Ten listeners, aged 20-92 years, with bilateral sensorineural hearing loss $(\mathrm{HL})$ participated in the study while attending a weeklong auditory rehabilitation workshop. They all gave full informed consent to complete the investigation that was approved by the University of Texas at Dallas Institutional Review Board. All had more than two years' experience with 
Table 1 Demographic Information Regarding Participants

\begin{tabular}{|l|l|l|l|l|l|l|}
\hline Participant & Age (years) & Gender & Technology & PTA right $(\mathrm{dB} \mathrm{HL})$ & PTA left (dB HL) & Onset of hearing loss \\
\hline 1 & 22 & Female & Bolero V90* & 57 & 55 & Congenital \\
\hline 2 & 22 & Female & Naida V90 SP & 62 & 63 & Congenital \\
\hline 3 & 92 & Female & Naida V90 SP & 48 & 47 & Acquired \\
\hline 4 & 78 & Female & Naida V90 SP & 28 & 43 & Acquired \\
\hline 5 & 85 & Female & Naida V90 UP & 60 & 53 & Acquired \\
\hline 6 & 83 & Female & Naida V90 SP & 60 & 42 & Acquired \\
\hline 7 & 22 & Female & Cochlear N6 & N/A & N/A & Congenital \\
\hline 8 & 52 & Female & Naida V90 UP* & 68 & 82 & Congenital \\
\hline 9 & 20 & Female & Naida V90 SP & 67 & 70 & Acquired \\
\hline 10 & 23 & Male & Cochlear N6 & N/A & N/A & Acquired \\
\hline
\end{tabular}

Note: PTA = pure-tone average in $\mathrm{dB} \mathrm{HL}$.

*Denotes the use of personal HA.

HAs or Cls, and half had more than five years' experience with remote microphone systems. Those with congenital losses reported unknown origin, and those with acquired losses reported etiologies including aging, genetic factors (\#9), or autoimmune inner ear disease (\#10). Participant criteria included the ability to repeat sentences presented in quiet with $100 \%$ accuracy and self-report of the ability to independently manage HA technology. As summarized in -Table 1, all participants wore bilateral behind-the-ear HAs, with two exceptions who wore CIs. The hearing loss in the better ear for the participants with HAs is shown in -Figure 1.

\section{Hearing Technology}

The wireless technology used in the study is shown in - Figure 2. Two Phonak wireless microphones were evaluated: the Roger Pen and the Roger Select microphone. All participants with hearing loss were fit with bilateral Phonak Naida HAs with two exceptions (\#1 and \#8). All aids were compatible with integrated Roger receivers (Roger 18 or 19).

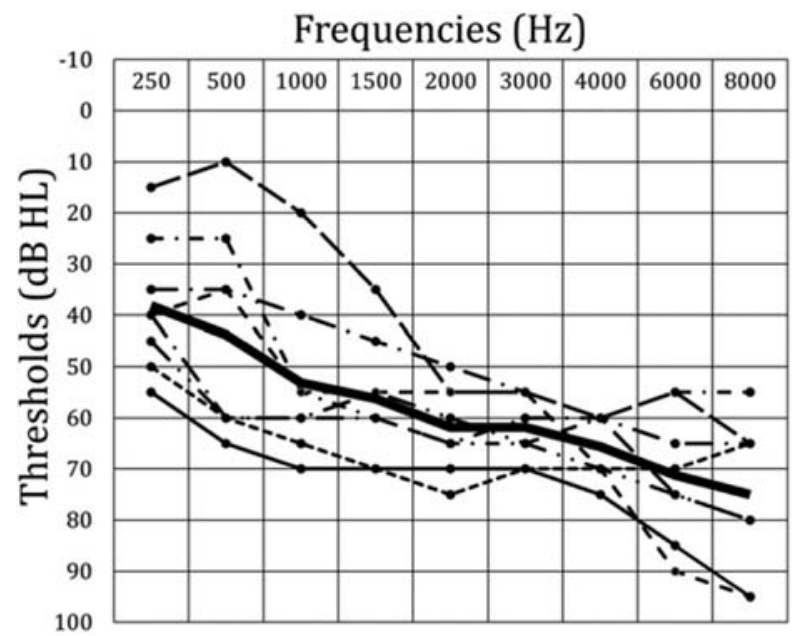

Fig. 1 Hearing thresholds of the better ear for participants with hearing loss. The average is represented by the bold black line.
All aids were programmed and verified with real-ear measures for NAL-NL1 targets (Byrne et $\mathrm{al}^{4}$ ). The impact of listening with a new amplification system for some of the listeners was considered minimal, given that the aid and settings were held constant for each listener across the listening conditions. Two participants (\#7 unilateral and \#10 bilateral) used their cochlear N6 implants with Roger $\mathrm{X}(02)$ receivers connected via euroadapters.

\section{Test Arrangement}

The experimental setup, as shown in - Figure 3, included five loudspeakers (Fostex 6302 B3E) arranged equidistance around a four-foot-diameter circular table. The test room was $15 \times 20$ feet and had carpet and acoustic tiles. The stimuli were presented from a laptop (Toshiba) connected to a sound board (Focusrite Scarlett 18i20) using Cubase software (Steinberg Media Technologies ${ }^{13}$ ). The competition was presented from a laptop (Toshiba) and two speakers (HDMX JAM).
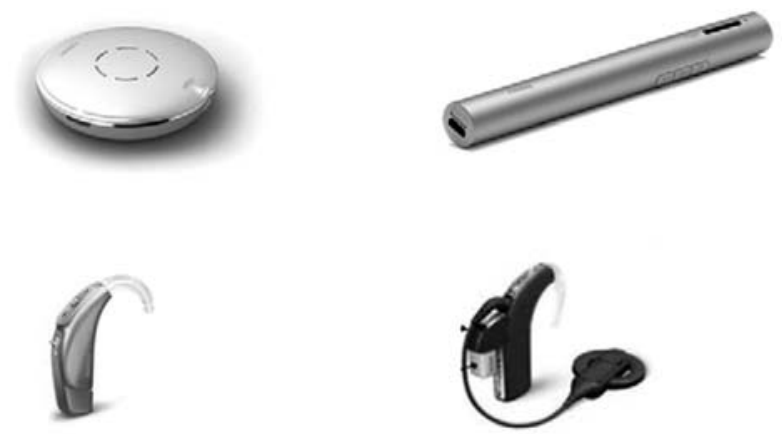

Fig. 2 Assistive technology used by the participants included Phonak Roger Select and Roger Pen microphones (top left and right) and Phonak Naida v90 SP/UP HAs with integrated Roger 18/19 receivers and N6 Cls with euroadapters and Roger $X$ receivers (bottom left and right). (Photos courtesy of Phonak) (This figure appears in color in the online version of this article.) 


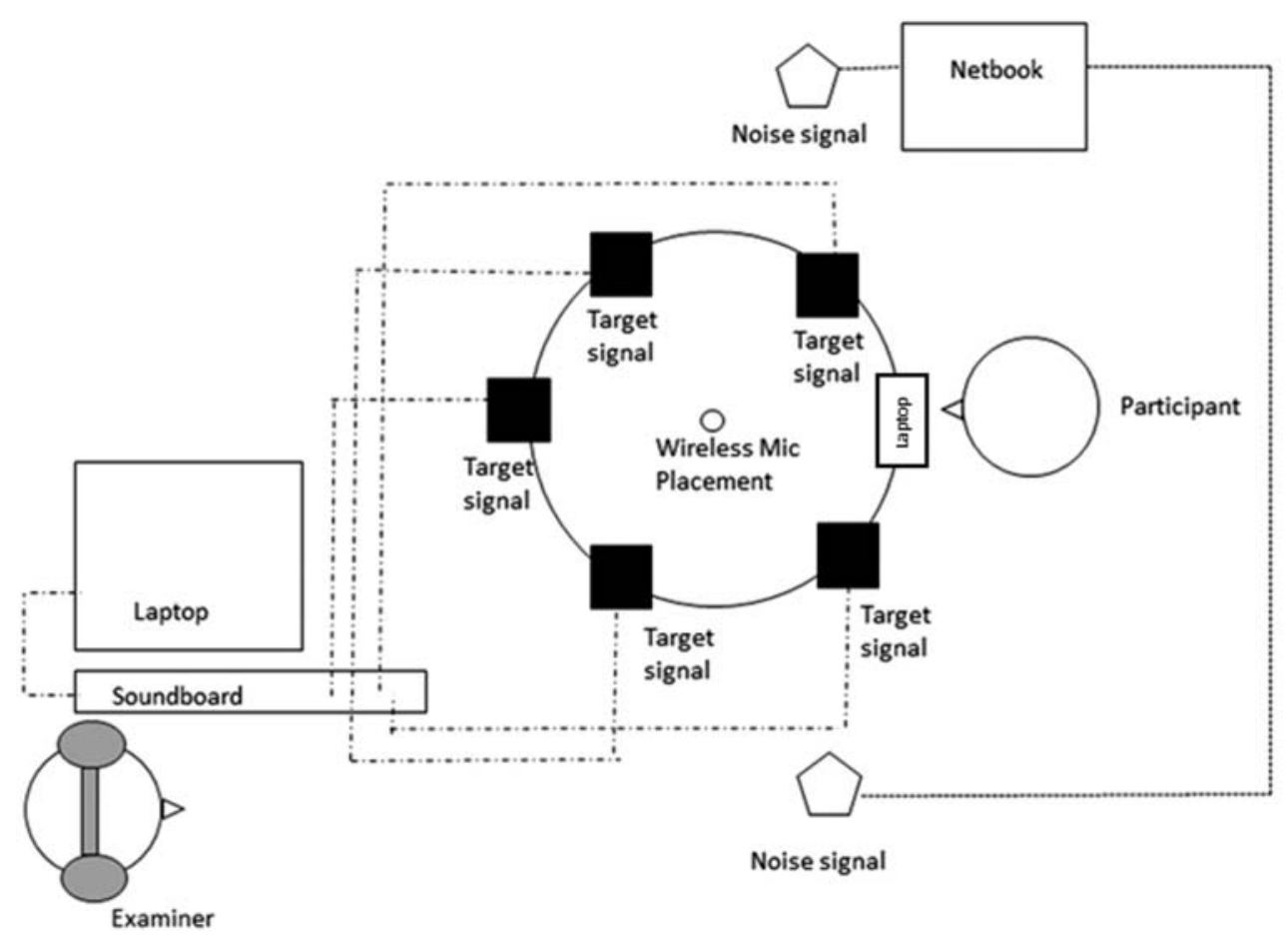

Fig. 3 Testing arrangement for evaluation of speech recognition in noise with wireless microphones placed in the center of the table.

\section{Stimuli}

Speech recognition in noise was measured using lists 1 through 12 of Hearing in Noise Test (HINT) sentences (Nilsson et $\mathrm{al}^{11}$ ), with a different list of 10 sentences randomly selected for each listening condition for each participant. The competition was a modified version of recorded restaurant noise downloaded from the Internet. Intensity spikes in the noise associated with plates clanging were removed using audio-editing software. Portions of the file were randomly concatenated to create a three-minute sample of noise. Both stimuli were calibrated at the location of the listener such that the speech was delivered at a constant 65-dBA level, whereas the noise was adjusted for the various conditions to result in $+5,0,-5$, and $-10 \mathrm{~dB}$ SNRs. To calibrate the stimulus level from the five loudspeakers around the table, a sentence was played repeatedly from each one and the volume level was adjusted independently to achieve the same sound pressure level at the location ofthe listener. To evaluate the performance when the signal level was increased as might be performed by a talker in a noisy restaurant, one additional SNR condition was completed at the highest noise in tensity, $75 \mathrm{dBA}$, with the signal presented at $70 \mathrm{dBA}$, or $-5 \mathrm{~dB}$ signal-to-noise ratio when signal is at $70 \mathrm{dBA}\left(\mathrm{S}_{70} \mathrm{NR}\right)$.

\section{Procedure}

A quasi-experimental, repeated-measures design was used to evaluate speech recognition performance across three listening conditions: $\mathrm{HA} / \mathrm{CI}$ alone, $\mathrm{HA} / \mathrm{CI}$ with Roger Pen in the omnidirectional mode, or $\mathrm{HA} / \mathrm{CI}$ with a Roger Select microphone in the adaptive directional mode. During the two-hour test session, the HINT sentences were presented randomly from one of the five speakers around the table, whereas the restaurant noise was presented from the two speakers at $45^{\circ}$ azimuth, 5 feet on either side of the listener.
All participants completed a practice list of ten sentences at $+5 \mathrm{~dB}$ SNR with Roger Pen technology before beginning the randomized conditions. A tablet with a keyboard was laid flat on the table in front of the listeners so that listeners could type their responses. Only four persons chose to verbally repeat the sentences, and the examiner typed the responses. To ensure scoring accuracy, all participants wore a Roger Pen microphone that transmitted to Roger Focus receivers worn by the examiner under circumaural headphones.

For each listening condition, the participants completed one randomly selected list of ten sentences presented at $65 \mathrm{dBA}$ in the lowest noise level ( $60 \mathrm{dBA},+5 \mathrm{~dB}$ SNR) and then progressed to the next more intense noise level if they heard at least one word in the first five sentences. The percentage of words correctly identified was determined for each list presented. When no responses were obtained for the first five sentences, a score of $0 \%$ correct was assigned and testing continued to the next more intense noise-level condition. The final condition was conducted for the three technology arrangements at the highest noise level, $75 \mathrm{dBA}$, and with the speech level increased to $70 \mathrm{dBA}$. Excluding the practice list, there were 11 HINT sentence lists used across the 15 listening trials ( 3 equipment arrangements $\times 5$ SNR levels). Therefore, each participant was presented four randomly selected lists a second time. In some instances, these were lists on which they were unable to hear any or very few words depending on the SNR/equipment arrangement.

\section{Results}

Percent correct scores obtained in the three technology conditions, $\mathrm{HA} / \mathrm{CI}$ alone, $\mathrm{HA} / \mathrm{CI}$ + Roger Pen, and $\mathrm{HA} / \mathrm{CI}+$ Roger Select microphone, are shown in -Figure 4. The Roger Select condition resulted in the best performance across noise levels 


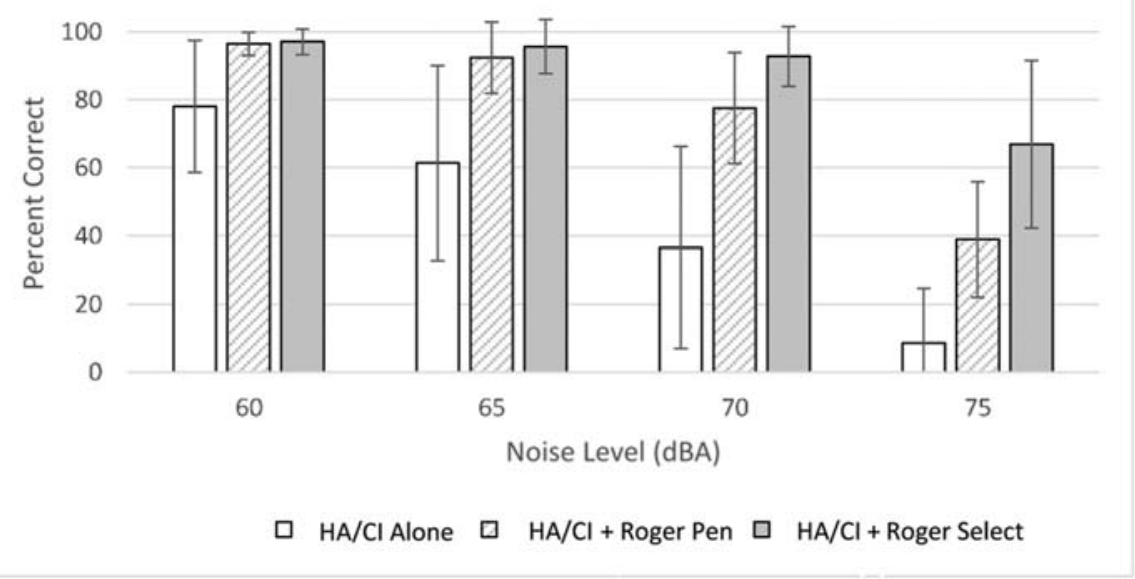

Fig. 4 Mean HINT sentence scores across noise levels for listeners with hearing impairment. Error bars represent \pm one SD.

$(\mathrm{M}=88.21 \%$, standard deviation $[\mathrm{SD}]=1.83)$ followed by the Roger Pen condition $(\mathrm{M}=76.31 \%, \mathrm{SD}=2.61)$ and then $\mathrm{HA} / \mathrm{Cl}$ alone condition $(\mathrm{M}=46.14 \%, \mathrm{SD}=3.53)$. As expected, performance across all technology conditions decreased as the noise level increased from $60(\mathrm{M}=90.48 \%, \mathrm{SD}=14.31)$ to $75 \mathrm{dBA}$ $(\mathrm{M}=38.15 \%, \mathrm{SD}=30.71)$.

Before statistical analysis, the scores were arcsine transformed to account for unequal variance in percent correct data (Sherbecoe and Studebaker ${ }^{12}$ ). A two-factor, repeated-measures analysis ofvariance was conducted for the four noise levels and three technology types (HA/CI alone, HA/CI + Roger Pen, and $\mathrm{HA} / \mathrm{CI}+$ Roger Select). There was a significant main effect of technology $\left[\mathrm{F}_{(3,108)}=52.48, p<0.0001\right]$ and noise level $\left[\mathrm{F}_{(2}\right.$, $\left.{ }_{108)}=65.58, p<0.0001\right]$, and a significant interaction between technology and noise level $\left[\mathrm{F}_{(6,108)}=2.47, p<0.05\right]$. As shown in - Table 2, this significant interaction was evaluated through follow-up post hoc analyses. To account for multiple comparisons, a Bonferroni-adjusted alpha value of 0.004 (0.05/12) was used. Results revealed significantly greater speech recognition performance for the Roger Select $(M=93.31 \%, S D=9.02)$ over the Roger Pen $(M=77.49 \%, S D=16.34)$ at the 70 -dBA noise level $\left[t_{(9)}=3.44, p<0.004\right]$. Performance with the Roger Select $(\mathrm{M}=66.88 \%, \mathrm{SD}=24.62)$ was also significantly better than that with the Roger Pen $(M=38.97 \%, S D=16.92)$ at the $75-d B A$ noise level $\left[t_{(9)}=3.62, p<0.004\right]$. Cohen's effect size value suggested a high practical significance $(d=1.19$ and 1.32 for 70 and 75 dBA noise levels, respectively). In addition, speech

Table 2 Values from Post Hoc Analysis of Pair-Wise Comparisons of Speech Recognition Scores

\begin{tabular}{|l|l|l|l|l|}
\hline Noise level (dBA) & 60 & 65 & 70 & 75 \\
\hline $\mathrm{HA} / \mathrm{Cl}$ vs. Roger Pen & $4.76^{*}$ & $4.81^{*}$ & $6.20^{*}$ & $8.70^{*}$ \\
\hline $\mathrm{HA} / \mathrm{Cl}$ vs. Roger Select & $3.45^{*}$ & $4.56^{*}$ & $6.87^{*}$ & $6.46^{*}$ \\
\hline Roger Pen vs. Roger Select & 0.40 & 1.06 & $3.44^{*}$ & $3.62^{*}$ \\
\hline
\end{tabular}

*Significance determined according to Bonferroni adjustment for multiple comparisons, resulting in $p<0.004$. recognition performance with the Roger Pen and the Roger Select was significantly better than performance with the $\mathrm{HA} / \mathrm{CI}$ alone at all four noise levels (all p's $<0.004$ ).

Because some listeners with hearing loss think that HAs alone are adequate in noisy environments, it was of interest to examine the actual benefit obtained with the two wireless microphone types compared with using just the $\mathrm{HA}$ or $\mathrm{CI}$ alone. The benefit in speech recognition in noise was determined by subtracting the $\mathrm{HA} / \mathrm{CI}$ alone score from each of the microphone scores. As shown in - Table 3, the greatest benefit (96.08\%) was achieved when using the Select microphone at the 75-dBA noise level for listener \#4. Across all listeners, the average benefit achieved with the Roger Select microphone increased from $19.01 \%$ to $58.29 \%$ as the noise level increased from 60 to $75 \mathrm{dBA}$.

The three listening conditions were also completed at the highest noise level, $75 \mathrm{dBA}$, but with the speech presented at $70 \mathrm{dBA}$, or $-5 \mathrm{~dB} \mathrm{~S}_{70} \mathrm{NR}$. This speech level was $5 \mathrm{~dB}$ more intense than that in the previous set of conditions to simulate how persons increase their vocal effort when in very noisy situations. As shown in -Table 4, the mean results were 22.83 ( $\mathrm{SD}=31.67), 68.23(\mathrm{SD}=24.17)$, and $84.09 \%(\mathrm{SD}=14.40)$ correct for $\mathrm{HA} / \mathrm{CI}$ alone, $\mathrm{HA} / \mathrm{CI}+$ Roger Pen, and $\mathrm{HA} / \mathrm{CI}+$ Roger Select conditions, respectively. The benefit achieved with each remote microphone technology relative to listening with $\mathrm{HAs} / \mathrm{CIs}$ alone is shown in = Figure 5 for this $-5-\mathrm{dB} \mathrm{S}_{70} \mathrm{NR}$ condition and for the previously reported -10 -dB signal-tonoise ratio when signal is at $65 \mathrm{dBA}$ condition for comparison. At this - 5-dB S ${ }_{70} \mathrm{NR}$, participants performed $61.26 \%(\mathrm{SD}=26.84)$ better with the Roger Select and $45.4 \%(S D=27.80)$ better with the Roger Pen microphone than with their HA/CI alone. A twoway, repeated-measures analysis of variance on the arcsinetransformed data obtained at $-5 \mathrm{~dB} \mathrm{~S}_{70} \mathrm{NR}$ and at $-10 \mathrm{~dB}$ signal-to-noise ratio when signal is at $65 \mathrm{dBA}$ revealed that significantly greater benefit was achieved with the Roger Select than the Roger Pen microphone $\left[F_{(1,36)}=6.33, p<0.05\right]$ but no significant effect of the SNR $\left[F_{(1,36)}=0.75, p>0.05\right]$ and no significant interaction $\left[F_{(1,36)}=0.33, p>0.05\right]$. 
Table 3 Percent Benefit Achieved in Speech Recognition When Using Roger Pen and Roger Select Compared with Ear-Level Technology Alone across Four Noise Levels (Benefit = Wireless Microphone Score - HA/Cl Alone Score)

\begin{tabular}{|l|l|l|l|l|l|l|l|l|}
\hline \multirow{2}{*}{ Noise level (dBA) Participant } & \multicolumn{2}{|l|}{$6 \mathbf{6 5}$} & \multicolumn{2}{l|}{70} & \multicolumn{2}{l|}{75} \\
\cline { 2 - 9 } & Pen & Select & Pen & Select & Pen & Select & Pen & Select \\
\hline $1^{*}$ & 3.85 & 0.00 & 0.00 & 13.21 & 41.51 & 41.51 & 42.59 & 86.27 \\
\hline 2 & -0.11 & 1.82 & 16.99 & 16.99 & 31.11 & 54.78 & 43.40 & 80.70 \\
\hline 3 & 21.86 & 18.75 & 60.37 & 61.04 & 70.88 & 65.17 & 23.08 & 43.13 \\
\hline 4 & 18.18 & 14.54 & 24.40 & 22.55 & 36.02 & 45.28 & 39.62 & 96.08 \\
\hline 5 & 54.71 & 62.26 & 52.24 & 83.02 & 49.02 & 90.91 & 24.07 & 54.39 \\
\hline 6 & 38.99 & 44.44 & 70.59 & 72.55 & 48.60 & 85.25 & 31.48 & 74.51 \\
\hline 7 & 19.68 & 19.71 & 14.81 & 22.65 & 67.04 & 73.49 & 11.77 & 57.89 \\
\hline 8 & 9.69 & 11.57 & 26.06 & 7.84 & 13.81 & 24.57 & 29.41 & -1.71 \\
\hline 9 & 3.92 & 1.95 & 1.89 & -0.03 & 3.43 & 5.43 & 25.54 & 49.17 \\
\hline 10 & 12.99 & 15.10 & 42.59 & 42.59 & 47.73 & 75.54 & 32.88 & 42.49 \\
\hline Mean & 18.38 & 19.01 & 30.99 & 34.24 & 40.92 & 56.19 & 30.38 & 58.29 \\
\hline SD & 17.08 & 19.88 & 24.38 & 29.90 & 21.11 & 27.90 & 9.86 & 28.19 \\
\hline
\end{tabular}

*Denotes the use of personal aids for the study.

Table 4 Speech Recognition Percent Correct Scores in Three Technology Conditions in 75 dBA Noise with Speech Presented at -10 and $-5 \mathrm{~dB}$ SNRs

\begin{tabular}{|c|c|c|c|c|c|c|}
\hline & \multicolumn{2}{|l|}{$\mathrm{HA} / \mathrm{Cl}$ only } & \multicolumn{2}{|c|}{$\mathrm{HA} / \mathrm{Cl}+$ Roger Pen } & \multicolumn{2}{|c|}{$\mathrm{HA} / \mathrm{Cl}+$ Roger Select } \\
\hline & $-10 \mathrm{~dB}$ SNR & $-5 \mathrm{~dB}$ SNR & $-10 \mathrm{~dB}$ SNR & $-5 \mathrm{~dB}$ SNR & $-10 \mathrm{~dB}$ SNR & $-5 \mathrm{~dB}$ SNR \\
\hline 1 & 0.00 & 37.25 & 42.59 & 98.11 & 86.27 & 100.00 \\
\hline 2 & 0.00 & 60.78 & 43.40 & 88.68 & 80.70 & 96.08 \\
\hline 3 & 0.00 & 0.00 & 23.08 & 28.30 & 43.13 & 84.31 \\
\hline 4 & 0.00 & 3.64 & 39.62 & 78.43 & 96.08 & 79.25 \\
\hline 5 & 0.00 & 0.00 & 24.07 & 25.93 & 54.39 & 62.75 \\
\hline 6 & 0.00 & 0.00 & 31.48 & 80.00 & 74.51 & 88.68 \\
\hline 7 & 3.92 & 0.00 & 15.69 & 70.17 & 61.81 & 56.60 \\
\hline 8 & 23.53 & 39.62 & 52.94 & 63.64 & 21.82 & 84.31 \\
\hline 9 & 49.01 & 87.03 & 74.55 & 84.91 & 98.18 & 94.34 \\
\hline 10 & 9.43 & 0.00 & 42.31 & 64.15 & 51.92 & 94.55 \\
\hline Mean & 8.59 & 22.83 & 38.97 & 68.23 & 66.88 & 84.09 \\
\hline SD & 16.06 & 31.67 & 16.92 & 24.17 & 24.62 & 14.40 \\
\hline
\end{tabular}

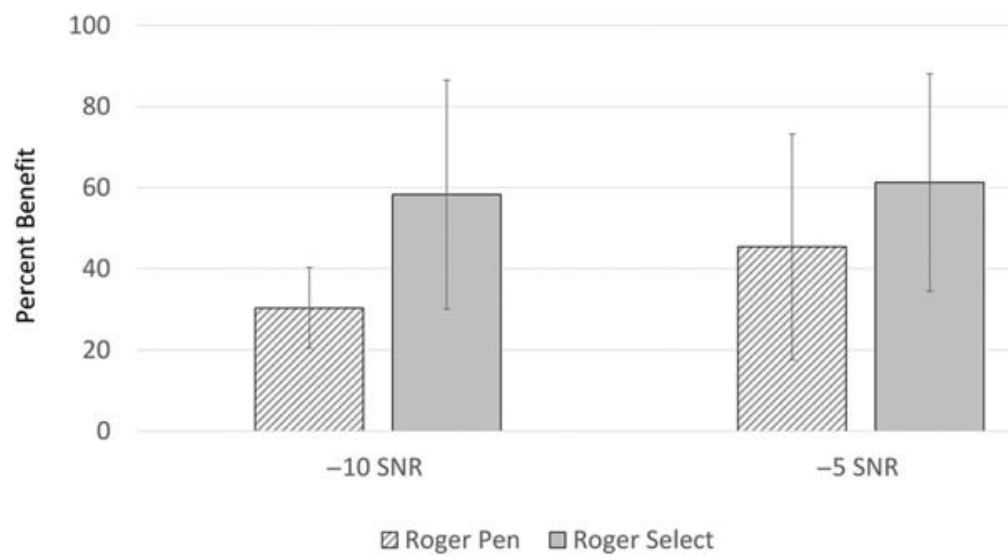

Fig. 5 Average benefit in HINT sentence scores for listeners with hearing impairment when using Roger Pen and Roger Select microphones for -10- and $-5-d B$ SNR listening conditions. Error bars represent \pm one SD. 


\section{Discussion}

The results of this investigation add to the evidence of the substantial benefits in speech recognition in noise provided by remote microphone technology. Documenting improvements in multitalker situations with adaptive directional microphone technology provides even greater motivation to determine communication challenges ofindividuals with hearing loss and consider all possible technology solutions. As with most research with persons with hearing loss, there was considerable variability in the degree of improvement, ranging from no benefit in some noise levels to a maximum benefit of $96.08 \%$ achieved by listener \#4 in the 75-dBA noise condition. All but two participants achieved their greatest benefit with the Roger Select microphone in either the 65- or 75-dBA noise condition. These two individuals, \#3 and \#8, however, did achieve their maximum benefit with Roger Select when tested in the $-5-\mathrm{dB} \mathrm{S}_{70} \mathrm{NR}$ condition (84.31\% and $44.69 \%$ improvements relative to $\mathrm{HA}$ alone, respectively). There were no observable differences in performance based on factors such as length of experience with remote microphone technology.

It is noteworthy that the wireless microphone technology allowed persons with hearing loss to communicate in noise with accuracy ranging from $56.60 \%$ to $100 \%$ in the most challenging noise level used in the current study. This was achieved with presentation of sentences from one of five loudspeakers with no clues that could facilitate orientation to the loudspeaker like might occur in the real-world situations. Furthermore, when attention can be focused on a topic ofconversation and the listener could select the directional pattern to focus a microphone toward a talker of interest, the perceived benefit by the listener would likely be much greater than represented by these scores obtained for unrelated sentences (Başkent et $\mathrm{al}^{1}$ ).

These results should be considered relative to the controlled testing arrangement. Although intended to simulate a group dining experience, the noise was relatively homogenous and delivered from a single sound source that was split to two speakers placed beside the listener. In a real-world setting with greater distractions and multiple talker characteristics, the speech recognition task could be more challenging. The noise in an actual restaurant would be more diffuse and potentially more variable. In addition, dining experiences are influenced by many aspects of ambient music that is often played at high levels, which further complicates the listening challenge (Biswas et $\mathrm{al}^{2}$ ). These factors may be offset, however, by the ability of listeners to use context and speech-reading cues.

The clinical implications of these findings could significantly impact the rehabilitative component of audiological services. Many persons with hearing loss have withdrawn from social interaction, particularly in group settings. Allowing individuals to experience the benefits of remote microphone technology may facilitate their acceptance of personal devices and result in improved quality of life. Given the multiple ways that personal assistive technology can be interfaced with other devices such as smartphones or music players, the use of remote microphone technology could add significant value to the range of benefits and should be considered when individuals report communication challenges in noise.

\section{Summary}

The use of a wireless microphone transmitting to a receiver coupled to HAs or CIs provided up to $45 \%$ (Roger Pen) and $61 \%$ (Roger Select) benefit on average in sentence recognition over listening with $\mathrm{HA} / \mathrm{CI}$ alone in a simulated noisy restaurant setting for participants with hearing loss. The use of these microphones, especially the Roger Select, would significantly reduce the challenges faced by persons with hearing loss in social settings when there are multiple talkers.

\section{Abbreviations}

$\begin{array}{ll}\text { CI } & \text { cochlear implant } \\ \text { HA } & \text { hearing aid } \\ \text { HINT } & \text { Hearing in Noise Test } \\ \text { SD } & \text { standard deviation } \\ \text { SNR } & \text { signal-to-noise ratio } \\ \text { S70NR } & \text { signal-to-noise ratio when signal is at 70 dBA }\end{array}$

Conflict of Interest

None declared.

\section{Acknowledgments}

The author thanks Phonak for providing the wireless technology and the participants in the Summer Intensive Auditory Revitalization Conference for their involvement. Appreciation is expressed to Dr. Rachel Nielsen for statistical consulting.

\section{References}

1 Baskent D, Clarke J, Pals C, Benard MR, Bhargava P, Saija J, Sarampalis A, Wagner A, Gaudrain E. Cognitive compensation of speech perception with hearing impairment, cochlear implants, and aging: how and to what degree can it be achieved? Trends in Hear 2016;20:1-16

2 Biswas D, Lund K, Szocs C. Sounds like a healthy retail atmospheric strategy: effects of ambient music and background noise on food sales. J Acad Mark Sci 2019;47:33-55

3 Boothroyd A. Hearing aid accessories for adults: the remote FM microphone. Ear Hear 2004;25:22-33

4 Byrne D, Dillon H, Ching T, Katsch R, Keidser G. NAL-NL1 procedure for fitting non-linear hearing aids: characteristics and comparisons with other procedures. J Am Acad Audiol 2001; $12: 3751$

5 De Ceulaer G, Bestel J, Mülder H, Goldbeck F, de Varebeke S, Govaerts P. Speech understanding in noise with the Roger Pen, Naida CIQ70 processor, and integrated Roger 17 receiver in a multi-talker network. Eur Arch Oto-Rhino-Laryngol 2016;273:1107-1114

6 Gigandet X, Fulton B, Smith C. Roger multibeam technology enhancing the group listening experience. Phonak Insight. 2018. https://www.phonakpro.com/au/en/products/wireless-accessories/roger-select/evidence-roger-select.html. Accessed June 1, 2019

7 Hawkins DB. Comparisons of speech recognition in noise by mildly-to-moderately hearing-impaired children using hearing aids and FM systems. J Speech Hear Disord 1984;49:409-418 
8 Kochkin S. MarkeTrak VIII: customer satisfaction with hearing aids is slowly increasing. Hear J 2010;63:11-19

9 Land V, Thibodeau L. Accuracy of speech recognition in a fivespeaker array using a hearing assistive device. Indianapolis, IN; 2017

10 Lewis M, Crandell C, Valente M, Horn J. Speech perception in noise: directional microphones versus frequency modulation (FM) systems. J Am Acad Audiol 2004;15:426-439

11 Nilsson M, Soli S, Sullivan J. Development of the Hearing in Noise Test for the measurement of speech reception threshold in quiet and in noise. J Acoust Soc Am 1994;95:1085-1099

12 Sherbecoe RL, Studebaker GA. Supplementary formulas and tables for calculating and interconverting speech recognition scores in transformed arcsine units. Int J Audiol 2004;43: $442-448$

13 Steinberg Media Technologies. Cubase Element 8. Hamburg, Germany; 2015. https://www.steinberg.net/en/support/downloads/cubase_8.html. Accessed May 1, 2015

14 Thibodeau L. Benefits of adaptive FM systems on speech recognition in noise for listeners who use hearing aids. Am J Audiol 2010; 19:1-10

15 Thibodeau L. Comparison of speech recognition with adaptive digital and FM remote microphone hearing assistance technology by listeners who use hearing aids. Am J Audiol 2014; 23:201-211 